\title{
Centrifuge experimental study on instability of seabed stratum caused by gas hydrate dissociation
}

\author{
X.H. Zhang ${ }^{\text {a,* }}$, X.B. Lu $^{\text {a }}$, Y.H. Shi ${ }^{\text {b }}$, Z. Xia ${ }^{\text {b }}$, W.T. Liu ${ }^{\text {b }}$ \\ a Institute of Mechanics, Chinese Academy of Sciences, Beijing 100190, China \\ ${ }^{\mathrm{b}}$ Marine Environmental and Engineering Geological Survey, Guangzhou Marine Geological Survey, Guangzhou 510075, China
}

\section{A R T I C L E I N F O}

\section{Article history:}

Received 15 September 2014

Accepted 6 June 2015

Available online 26 June 2015

Keywords:

Gas hydrate

Stratum instability

Centrifuge test

Dissociation zone

Pore fluid pressure

\begin{abstract}
A B S T R A C T
Gas hydrate $(\mathrm{GH})$ is a kind of solid energy resource with huge reserve. Stratum instability (such as marine landslide) may be caused by hydrate dissociation due to the softening of stratum and the buildup of excess pore pressure. In this paper, a centrifuge experiment was conducted to study the evolution of stratum instability during hydrate dissociation. The hydrate dissociation zone expands from the heating source in hydrate bearing sediment (HBS). The pore fluid pressure increases significantly accompanying the slow seepage and soil layer's softening and deformation. Large horizontal displacements and vertical displacements can be observed and increase with the expansion of hydrate dissociation zone. The results can be used for the verification of numerical simulations and a reference for engineering design.
\end{abstract}

c 2015 Elsevier Ltd. All rights reserved.

\section{Introduction}

Gas hydrate is regarded as a strategic energy resource and distributes extensively in permafrost and marine sediment under high pressure and low temperature conditions (Sloan, 1998; Kvenvolden and Lorenson, 2001). Sediments with gas hydrate have been obtained from many ocean and permafrost areas, such as Mallik in Canada (Winters et al., 2004), the continental slope in the northern South China Sea, Dongsha Sea and Qilian Mountains in China (Li et al., 2010; Zhu et al., 2010).

During hydrate exploitation and exploration or oil/gas recovery from reservoir overlaid by HBS, heat transfer from the wellbore or vertical pipes can lead to the surrounding GH dissociation and in turn the occurrence of seepage and deformation of stratum. The strength of HBS decreases greatly during/after GH dissociation due to the loose of adhesion among $\mathrm{GH}$ and soils and the generation of excess pore pressure (Winters et al., 2007; Waite et al., 2009; Lu et al., 2008). If GH dissociates in sediments with a relatively small permeability, excess pore pressure can be generated, which greatly decreases the effective stress and the strength of the stratum. This may cause geological and engineering hazards such as marine landslides, seabed subsidence, destruction of ocean platforms and oil pipes, and even gas blowouts (Sultan et al., 2004a, 2004b; Xu and Germanovich, 2006, 2007; Kwon et al., 2008; Zhang et al., 2011; Lu et al., 2013; Wang et al., 2009).

\footnotetext{
* Corresponding author.

E-mail address: zhangxuhui@imech.ac.cn (X.H. Zhang).
}

Some analytical models have been presented for stratum instability analysis during $\mathrm{GH}$ extraction which couples the heat conduction, GH dissociation, fluid flow and stratum deformation together. Marine landslide has been analyzed by limit equilibrium methods (Kimoto et al., 2010; Klar et al., 2010). However, few experimental evidences of failure patterns and mechanism have been reported.

Physical modeling plays an important role in solving geotechnical problems. Generally, it is concerned with replicating an event comparable to what might exist in the prototype. The model is often a reduced scale version of the prototype and that is particularly true when the physical events are similar and satisfy the scaling law. Centrifuge modeling is an effective tool for gravitational effects and large scale modeling in geotechnical materials because the stresses in the model and prototype are identical. A special feature of centrifuge modeling is its capability to reproduce both the strength and stiffness behavior of soils (Taylor, 1995). A test of one dimensional geo-mechanical and thermal responses of $\mathrm{CO}_{2}$ hydrate-bearing sands subjected to thermal simulation was conducted using a geotechnical centrifuge (Kwon et al., 2013). Coupled physical processes were observed in the tests containing heat transfer, softening of soils, pressure dissipation, gas migration. The results showed that heat transfer led to the hydrate dissociation accompanied by the pressure dissipation, the formation of excess pore pressure and the decrease of soil stiffness and shear strength. The deformation and fractures of soils during the test occurred indicating the possible stratum instability in future hydrate exploitation.

This paper aims to carry out a centrifuge model test to simulate the stratum instability triggered by GH dissociation. Firstly, controlling 
parameters were derived by dimensional analysis, hydrate sediments were dissociated by heating from a production well similar to hydrate thermal recovery and two different centrifuge accelerations were imposed to simulate the gravity effects. Secondly, the pore fluid pressure, temperature, soil deformation were monitored to investigate the physical mechanism of stratum instability during hydrate dissociation. Finally, the physical processes were analyzed by a decoupling method.

\subsection{Scaling law of the problem}

For centrifuge modeling, if an acceleration of $N$ times gravity $g$ is applied to a material with density $\rho$, then the vertical stress at depth $l_{m}$ in the model (indicated by subscript $m$ ) is given by $\sigma_{m}=\rho N g l_{m}$, and the prototype (indicated by subscript $p$ ) stress is $\sigma_{p}=\rho g l_{p}$. Thus for $\sigma_{m}=\sigma_{p}$, i.e. $\rho N g l_{m}=\rho g l_{p}$, we can obtain that: $l_{p}=N l_{m}$.

Then in centrifuge tests the soil stresses and deformations are similar with those of the full-scale prototype due to the proper modeling of body forces, which are important for the geotechnical problem, and for the capability of investigating undrained or partially drained conditions. The scaling laws for centrifugal experiments in this paper are shown in Table 1.

The problem contains two physical processes with different characteristic times: heat conduction and seepage.

The characteristic time of heat conduction (Tan, 2011) is

$t=\frac{\rho C l^{2}}{\lambda}$

The similarity relationship of the characteristic time of heat conduction is

$\frac{t_{m}}{t_{p}}=\frac{\rho_{m} C_{m} l_{m}^{2}}{\lambda_{m}} / \frac{\rho_{p} C_{p} l_{p}^{2}}{\lambda_{p}}=\frac{\lambda_{p} \rho_{m} C_{m}}{\lambda_{m} \rho_{p} C_{p}} \cdot \frac{l_{m}^{2}}{l_{p}^{2}}=\frac{\lambda_{p} \rho_{m} C_{m}}{\lambda_{m} \rho_{p} C_{p}} \cdot \frac{1}{N^{2}}$

where $\lambda$ is the coefficient of heat conduction, $t$ is time, $\rho$ is density of porous media, $C$ is the specific heat, and $l$ is the length related to the heat conduction path.

Here the expansion length of the heat-induced hydrate dissociation zone along the slope is considered as the hydraulic length for the seepage flow scaling. Hence, the characteristic length is the same with the heat conduction. The characteristic time of seepage can be deduced as follows (Taylor, 1995). According to Darcy's law for seepage flow:

$v=k \cdot i$

where $v, k, i$ represent superficial velocity of seepage flow, the coefficient of permeability, hydraulic gradient, respectively.

The relationship between the coefficient of permeability and intrinsic permeability can be expressed as:

$k=\frac{\rho g K}{\mu}$

where $K$ and $\mu$ represent the intrinsic permeability and viscosity, respectively.

Table 1

Centrifugal experimental similarities.

\begin{tabular}{ll}
\hline Parameters & Similarities \\
\hline Length & $1 / N$ \\
Stress & 1 \\
Strain & 1 \\
Force & $1 / N^{2}$ \\
Acceleration & $N$ \\
Frequency & $N$ \\
\hline
\end{tabular}

The characteristic time of seepage can be expressed as:

$t=\frac{l}{v}=\frac{l}{k i}=\frac{\mu}{\rho K i} \cdot \frac{l}{g}$

The similarity relationship of the seepage between model and prototype is expressed as:

$\frac{t_{m}}{t_{p}}=\left(\frac{\mu}{\rho K i} \cdot \frac{l}{g}\right)_{m} /\left(\frac{\mu}{\rho K i} \cdot \frac{l}{g}\right)_{p}=\frac{\mu_{m}(\rho K i)_{p}}{\mu_{p}(\rho K i)_{m}} \cdot \frac{l_{m} g_{p}}{l_{p} g_{m}}=\frac{\mu_{m}(\rho K i)_{p}}{\mu_{p}(\rho K i)_{m}} \cdot \frac{1}{N^{2}}$

when the prototype material is adopted in physical modeling, and the temperature and pressure are the same also, the similarity relationships of heat conduction and seepage can be simplified as follows:

For heat conduction: $\frac{t_{m}}{t_{p}}=\frac{1}{N^{2}}$

For seepage: $\frac{t_{m}}{t_{p}}=\frac{1}{N^{2}}$

From the above analysis, the heat conduction and seepage were both accelerated by $N^{2}$ times in the centrifuge test, so the model test will present similar characteristics with prototype and the model test can reflect the practical conditions and phenomenon. The ratio between heat conduction and seepage can be expressed approximately as $\frac{\rho C l}{\lambda} / \frac{\mu}{\rho_{w} g K \sin \alpha} \approx 100$ ( $\alpha$ is the slope of the stratum), thus these two processes can be decoupled. Here, $\rho, \rho_{w}, C, \lambda, l, K, \mu, g, \alpha$ are taken values as $1700 \mathrm{~kg} / \mathrm{m}^{3}$ $1000 \mathrm{~kg} / \mathrm{m}^{3}, 2800 \mathrm{~J} / \mathrm{kg} / \mathrm{K}, 1.0 \mathrm{w} / \mathrm{m} / \mathrm{K}, 1 \mathrm{~m}, 10^{-15} \mathrm{~m}^{2}, 10^{-3} \mathrm{~Pa} \mathrm{~s}, 9.8 \mathrm{~m} /$ $s^{2}, 14^{\circ}$, respectively (The data are referred to Hong et al., 2003 and measured soil parameters in centrifuge tests).

\subsection{Test setups and model preparation}

Fig. 1a and b presents the test setup with a global system of coordinates $(X, Y, Z)$. The size of the model box is $60 \mathrm{~cm}$ in length, $35 \mathrm{~cm}$ in width and $40 \mathrm{~cm}$ in height. The vaseline was applied on the inner sides for reducing the side friction and heat insulation before in the test. Silty soil from the shallow layer of the South

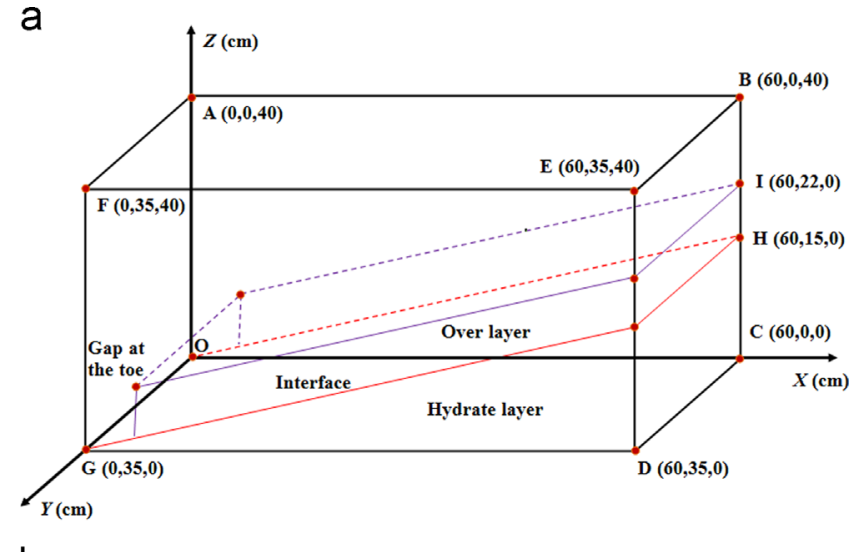

b

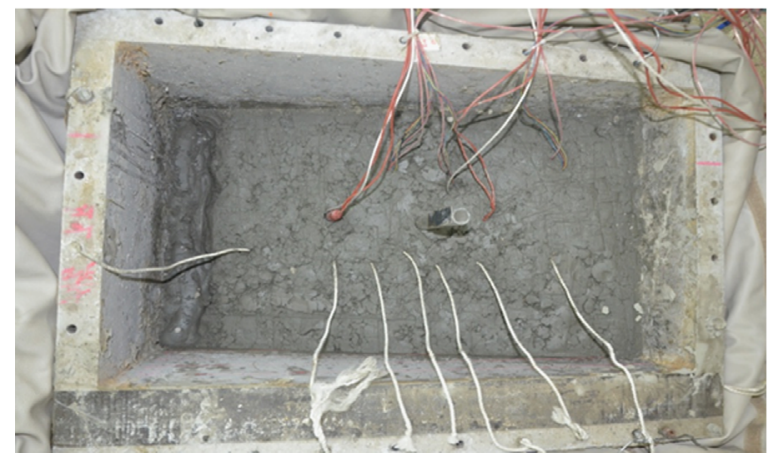

Fig. 1. Test setups. (a) Diagram of model set-ups with a global system of coordinates and (b) Physical model set-ups. 


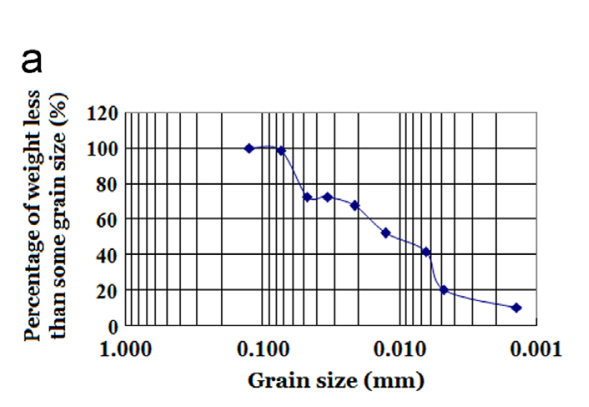

C

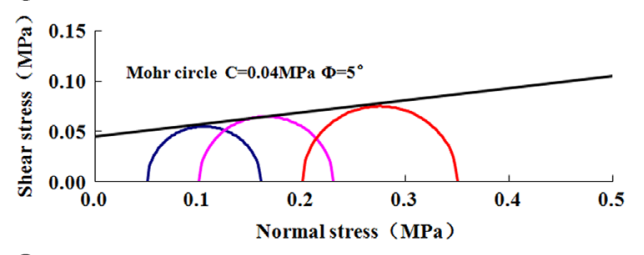

e

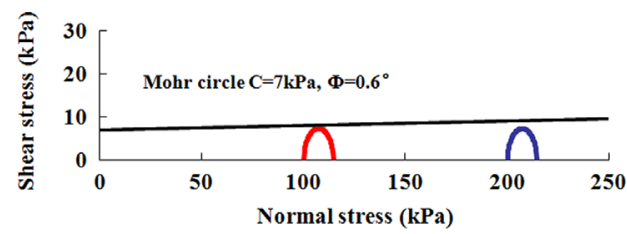

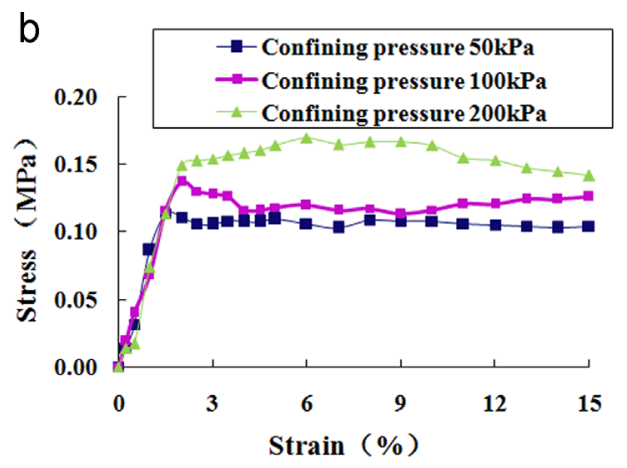

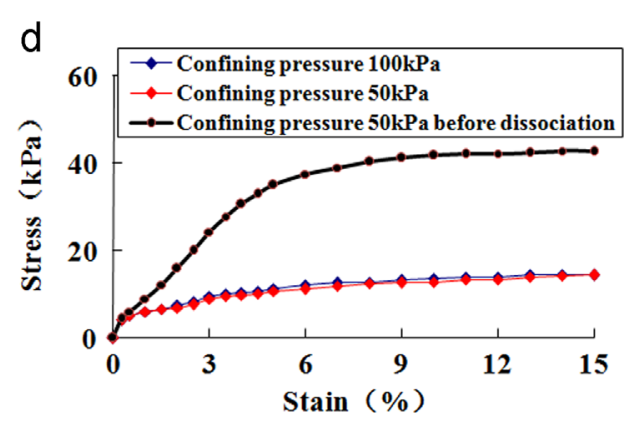

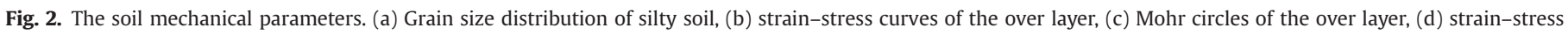
curves of the hydrate layer after hydrate dissociation, and (e) Mohr circles of the hydrate layer after hydrate dissociation.

China Sea over HBS was used whose specific gravity was about 2.75. The grain size distribution was as in Fig. 2a. Two layers i.e. hydrate layer and over layer, were prepared in the box with different dry densities and water contents. The hydrate layer was Firstly formed with a dry density of the clay layer $1.1 \mathrm{~g} / \mathrm{cm}^{3}$ and a porosity of $60 \%$.

Tetrahydrofuran (THF) hydrate bearing sediments are often adopted as a good substitute for methane hydrate in laboratory experiments due to the similarity in mechanical and thermal properties and a large volume of THF sediment can be synthesized more homogeneously, naturally and safely than synthesized methane hydrate sediment, since THF is completely miscible with water in all proportions and forms hydrate at $1 \mathrm{~atm}$ and $20{ }^{\circ} \mathrm{C}$ (Jones et al., 2007; Yun et al., 2007; Lee et al., 2007). THF hydratebearing sediment was then synthesized with a hydrate saturation of $20 \%$ and a water saturation of $80 \%$ in the pores under a temperature of $2{ }^{\circ} \mathrm{C}$. After the hydrate layer was prepared, the temperature was lowered to $-4{ }^{\circ} \mathrm{C}$ to keep the hydrate stable, then the over layer was set. The dry density of the over layer was $1.3 \mathrm{~g} / \mathrm{cm}^{3}$, the porosity was $52 \%$ and the water content was $34 \%$. The thicknesses of the hydrate layer and the over layer were $15 \mathrm{~cm}$ and $7 \mathrm{~cm}$, respectively. The slope of the over layer was $14^{\circ}$. The surfaces of the over layer and hydrate layer were parallel. A gap between the slope toe and the side wall with a length of $6 \mathrm{~cm}$ was left in order to measure the slippage of the slope due to the hydrate dissociation.

Triaxial tests under undrained and unconsolidated conditions were conducted to obtain the mechanical parameters. The internal friction angle and cohesion of over layer are $5^{\circ}$ and $40 \mathrm{kPa}$ under confining pressures of $50-200 \mathrm{kPa}$ (as in Fig. 2b). Two groups of THF hydrate soils similar to that in the centrifuge test were formed to model two states: non-dissociation and totally dissociation, for it is difficult to test in the triaxial chamber heating during load. After THF hydrate in the sample dissociates, solid hydrate in the pores transforms to THF-water solution and the sample became softened and almost saturated by fluid, which causes the

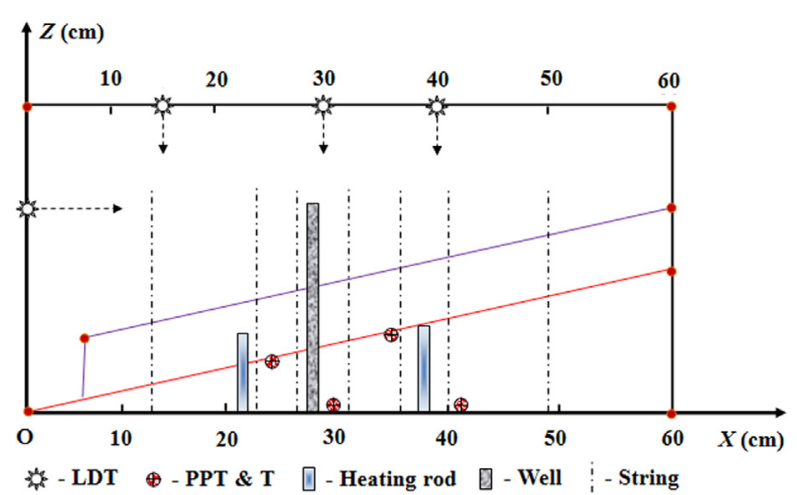

Fig. 3. Arrangement of heating rods, transducers, springs, and well.

Table 2

The coordinates of the heating rods, transducers, strings, and well.

\begin{tabular}{llll}
\hline Coordinates & $X(\mathrm{~cm})$ & $Y(\mathrm{~cm})$ & $Z(\mathrm{~cm})$ \\
\hline Heating rod 1 & 36.5 & 17.5 & $0-10$ \\
Heating rod 2 & 21.5 & 17.5 & $0-10$ \\
PPT1 \& T1 & 41 & 17.5 & 0 \\
PPT2 \& T2 & 35 & 17.5 & 8.7 \\
PPT3 \& T3 & 30 & 17.5 & 0 \\
PPT4 \& T4 & 24 & 17.5 & 6.0 \\
LDT1 & 40 & 17.5 & 40 \\
LDT2 & 0 & 17.5 & 30 \\
LDT3 & 30 & 17.5 & 40 \\
LDT4 & 15 & 17.5 & 40 \\
Well & 28.5 & 17.5 & $0-30$ \\
S1 & 49 & 16.5 & $0-40$ \\
S2 & 40 & 16.5 & $0-40$ \\
S3 & 35.5 & 16.5 & $0-40$ \\
S4 & 31 & 16.5 & $0-40$ \\
S5 & 27 & 16.5 & $0-40$ \\
S6 & 22.5 & 16.5 & $0-40$ \\
S7 & 13 & 16.5 & $0-40$ \\
\hline
\end{tabular}


stress-strain curves to be little difference under confining pressures of $50 \mathrm{kPa}$ and $100 \mathrm{kPa}$ And the internal friction angle and cohesion of the soil to decrease to be $0.6^{\circ}$ and $7 \mathrm{kPa}$, respectively. Before the hydrate dissociation, the change in shear strength with temperature is small. After the complete hydrate dissociation, the shear strength reduces to about $1 / 6$ that of hydrate bearing soil (as in Fig. 2c) and the compression modulus was 1.6 MPa.

Two heating rods 1, 2 were placed in the hydrate layer with a length of $10 \mathrm{~cm}$. The heating rods are set to reach $70{ }^{\circ} \mathrm{C}$. Transducers for measurement of pore fluid pressure, temperature and displacement were located as shown in Fig. 3. Pore fluid pressure transducers (PPT) ranged from $0 \mathrm{MPa}$ to $0.7 \mathrm{MPa}$, temperature

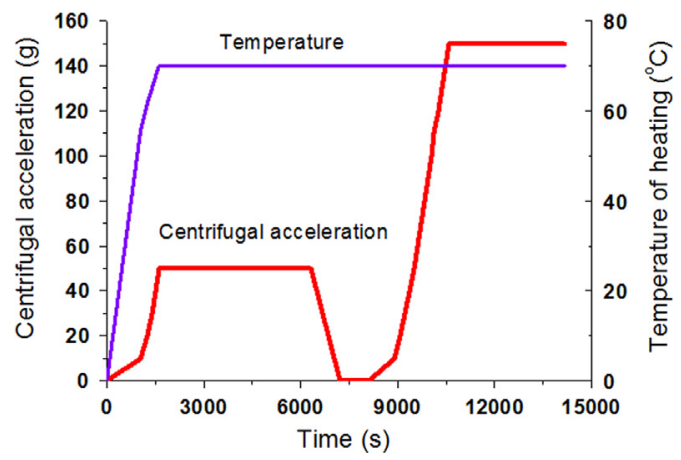

Fig.4. Heat source heating and centrifuge acceleration vs. time.

Table 3

The important time periods during the centrifuge test.

\begin{tabular}{lllllll}
\hline Centrifuge test process & Stage I & & \multicolumn{2}{c}{ Manual measurement } & \multicolumn{2}{l}{ Stage II } \\
\cline { 2 - 3 } & $1-50 \mathrm{~g}$ & $50 \mathrm{~g}$ & $50-1 \mathrm{~g}$ & $1-150 \mathrm{~g}$ & $150 \mathrm{~g}$ \\
\hline Time period (s) & 1300 & 4696 & 1800 & 2400 & 3605 \\
\hline
\end{tabular}
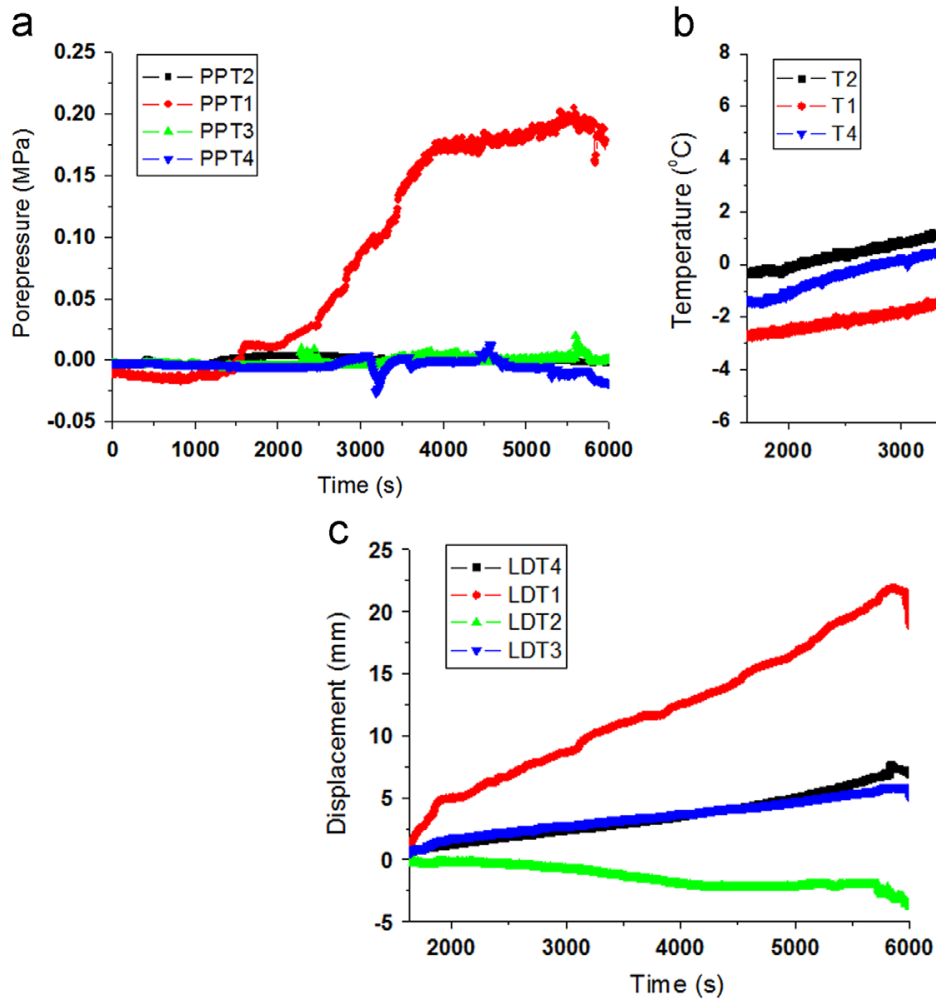

\subsection{Test procedures} deformation. transducers $(T)$ ranged from $-30{ }^{\circ} \mathrm{C}$ to $100{ }^{\circ} \mathrm{C}$, and laser displacement transducers (LDT) ranged from $0 \mathrm{~mm}$ to $50 \mathrm{~mm}, 120 \mathrm{~mm}$ and $270 \mathrm{~mm}$, respectively. The seven strings were set to mainly measure the horizontal displacements along the depth and so to determine the sliding face while the laser transducers were set to measure the vertical displacement at the surface of the over layer only. After the centrifuge test, the manual measurements of both horizontal and vertical displacement were referred to immovable labels at the side of the model box. The coordinates of the heating rods, transducers, strings, and well are listed in Table 2.

When the sample was prepared, the model box was placed in the basket of the centrifuge and the heating rods began to work. The centrifugal acceleration began to rise to reach $50 \mathrm{~g}$ accompanying the temperature of the heating source got to $70{ }^{\circ} \mathrm{C}$, Fig. $4 \mathrm{a}$ presents the development of centrifuge acceleration and temperature of heating source with time.

Two stages were conducted in the centrifuge test, and the time periods were presented in Table 3. In Stage I and Stage II, the maximum gravitational acceleration was $50 \mathrm{~g}$ and $150 \mathrm{~g}$, the corresponding prototype length of the stratum was $30 \mathrm{~m}$ and $90 \mathrm{~m}$, the thickness of the over layer was $3.5 \mathrm{~m}$ and $10.5 \mathrm{~m}$, and the maximum thickness of the hydrate layer was $7.5 \mathrm{~m}$ and $22.5 \mathrm{~m}$, respectively. The de-acceleration process and manual measurement both continued $15 \mathrm{~min}$. During the de-acceleration process, although the soil stress changed with the acceleration, the ratio of the characteristic times of heat conduction and fluid seepage kept the same as that of Stage I and Stage II. The seepage will become slow but little effects of the total consolidation degree since the most of it has finished before de-acceleration. Meanwhile a slow and small expansion of hydrate dissociation zone relative to the whole process had little effect to the fluid seepage and soil

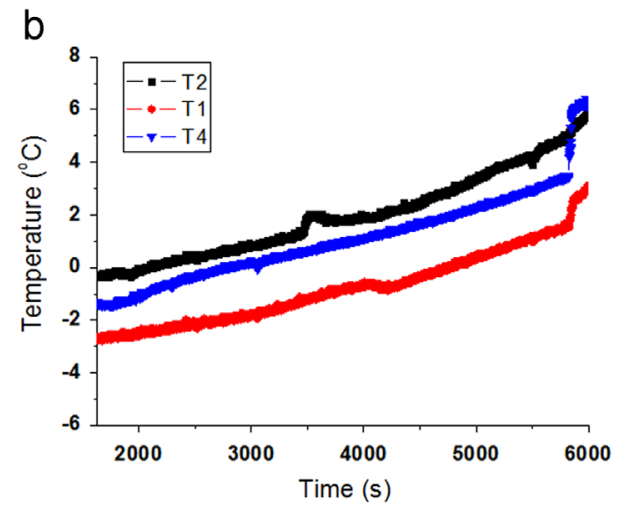

Fig. 5. The evolution of pore pressure, temperature and displacement in Stage I. (a) Pore pressure, (b) temperature, and (c)displacement. 

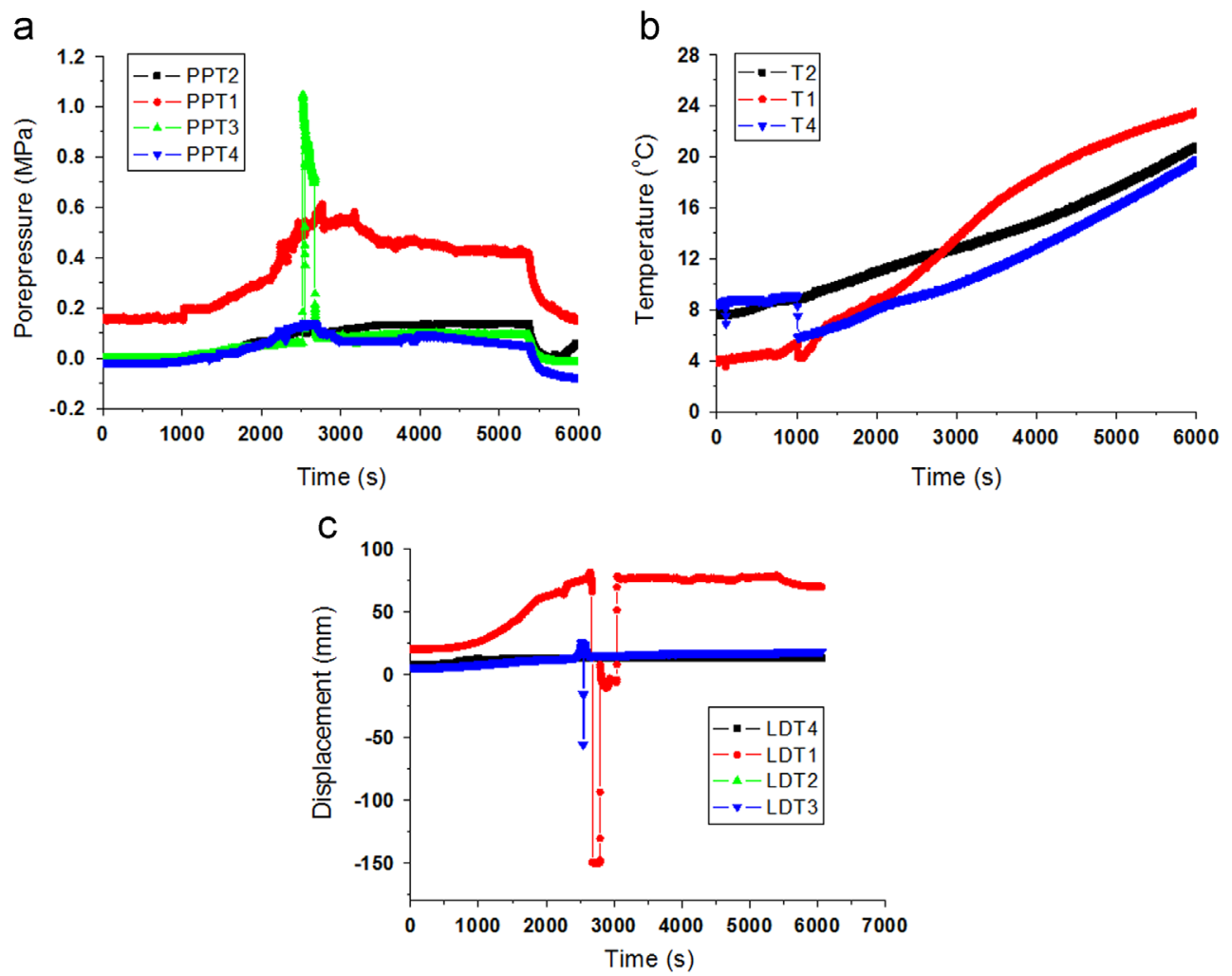

Fig. 6. The evolution of pore pressure, temperature and displacement in Stage II. (a) Pore pressure, (b) temperature, and (c) displacement.

\subsection{Test results}

Figs. 5 and 6 show the evolution of the pore fluid pressure, temperature and displacement. It is noted that all the following displacements are in model scale. In Fig. 5, the pore fluid pressure measured by PPT1 increases to $0.20 \mathrm{MPa}$ and then keeps stable (Initially, fluid connectivity is weak near the pressure transducers because pores are filled with hydrate. Thus the measured pressure is zero. The pore fluid pressure increases with the surrounding hydrate dissociates). PPT2, PPT3 and PPT4 showed zero or negative pressure in Stage I. The preliminary explanation is that the heat supply is less than that required for hydrate dissociation around the transducers and the fluid seepage path to the transducers are blocked. The maximum changes of the temperature and vertical displacement measured by T1, T2, T4 and LDT1, LDT2, LDT3, LDT4 were $3.2^{\circ} \mathrm{C}, 6.4{ }^{\circ} \mathrm{C}, 0.8^{\circ} \mathrm{C}$ and $15 \mathrm{~mm},-3 \mathrm{~mm}, 5 \mathrm{~mm}, 7 \mathrm{~mm}$, respectively. The leap of the temperature may be caused by the movement of transducers in the stratum. In Fig. 6, the pore fluid pressures measured by PPT1, PPT2, PPT3 and PPT4 are 0.57 MPa, $0.10 \mathrm{MPa}, 1.10 \mathrm{MPa}$ and $0.10 \mathrm{MPa}$, respectively. The maximum changes of the temperature and vertical displacement measured by T1, T2, T4 and LDT1, LDT2, LDT3, LDT4 are $19.8^{\circ} \mathrm{C}, 13.4{ }^{\circ} \mathrm{C}, 1.2^{\circ} \mathrm{C}$ and $50 \mathrm{~mm}, 55 \mathrm{~mm},-40 \mathrm{~mm}, 5 \mathrm{~mm}$, respectively. The test results show the decoupling of temperature and pressure development: The temperature increases while the pressure keeps as constants when it achieves the maximum value. The vertical displacement of the stratum increases gradually. The well inclined with the movement of the stratum. One or two leaps occurred in the displacements because of the cracking near the measured point.

Fig. 7 presents the vertical displacement at the surface of the stratum measured by a ruler after test. The maximum vertical displacement was $0.3 \mathrm{~cm}$ (This was smaller than that of the displacement transducers due to those lasers measured the depth of the cracks) in Stage I and $5.0 \mathrm{~cm}$ in Stage II. In Stage I, the over layer presents little slippage and the slope keeps constant, only a few local

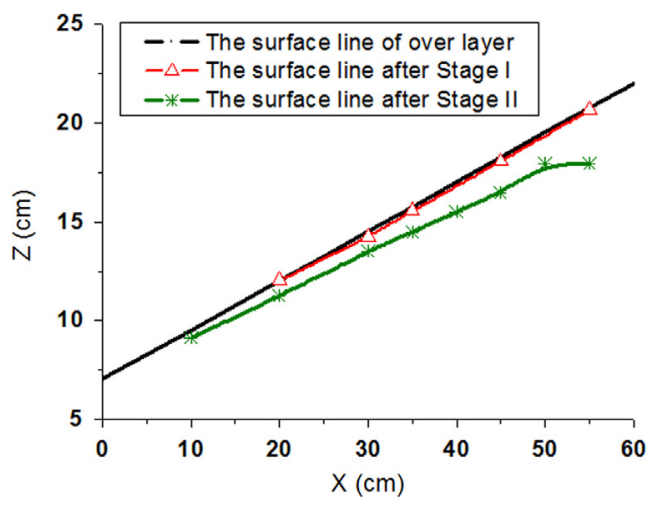

Fig. 7. Vertical displacement of the soil layers measured after testing.

circular cracks around the heating rod can be observed. But in Stage II, many large curved cracks occur in the over layer as in Fig. 8. The formation and development of the cracks are related to the slippage and the mass transfer. The slope decreases to $9^{\circ}$ from $14^{\circ}$ after the test, meanwhile the well inclined seriously $25^{\circ}$ from the vertical line.

From Fig. 9a and b, in Stage I, the horizontal displacements at the surface of the over layer were measured referring to the initial baseline set at the sides of the model box. The displacements were as follows: S1-0.5 cm, S2-0.5 cm, S3-1.0 cm, S4-0.5 cm, S5-1.0 cm, $\mathrm{S} 6-1.0 \mathrm{~cm}$, and $\mathrm{S} 7-1.5 \mathrm{~cm}$. The length of the dissociation zone is $8.5 \mathrm{~cm}$, and the radius of the crack zone is $18.5 \mathrm{~cm}$. In Stage II, the horizontal displacements measured by the strings at the surface of the over layer are as follows: $\mathrm{S} 1-4.0 \mathrm{~cm}, \mathrm{~S} 2-3.5 \mathrm{~cm}, \mathrm{~S} 3-4.0 \mathrm{~cm}, \mathrm{~S} 4-$ $4.0 \mathrm{~cm}, \mathrm{~S} 5-4.0 \mathrm{~cm}, \mathrm{~S} 6-5.5 \mathrm{~cm}$, and $\mathrm{S} 7-5.0 \mathrm{~cm}$. The inflection points of the strings are connected to indicate the sliding surface in Fig. 9a. Through the section excavation of the stratum is with a little disturbance, the height distributions of the over layer and the hydrate layer are obtained after test. In Stage II, the obvious sliding in the stratum can be observed. It should be noted that 
a

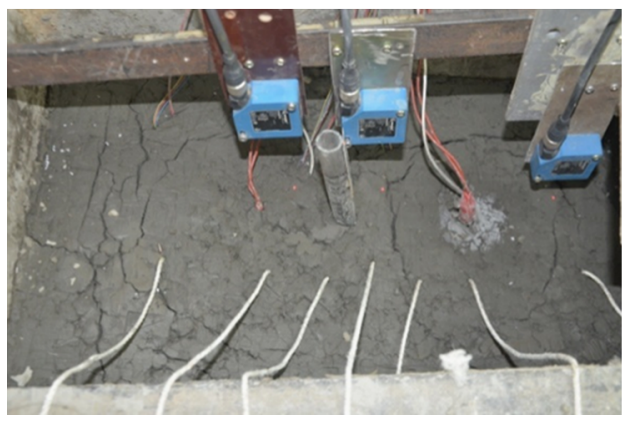

$b$

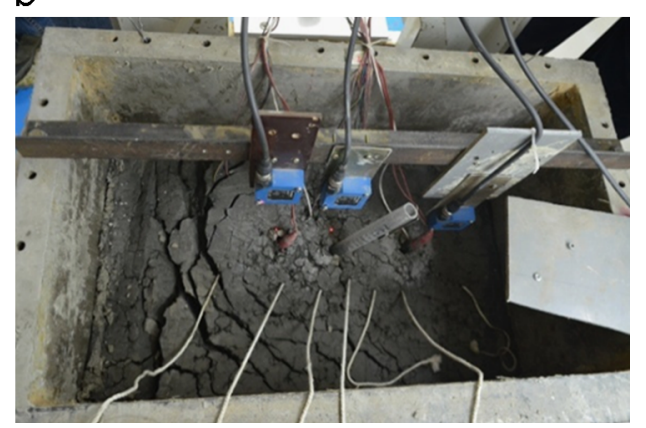

Fig. 8. Cracks after centrifuge testing. (a) Stage I and (b) Stage II.

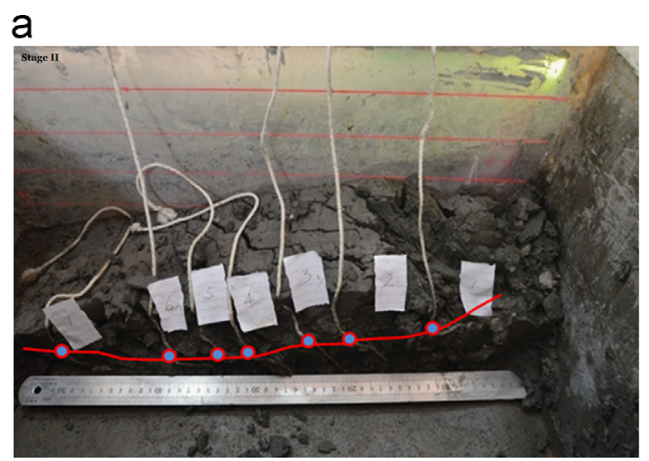

b
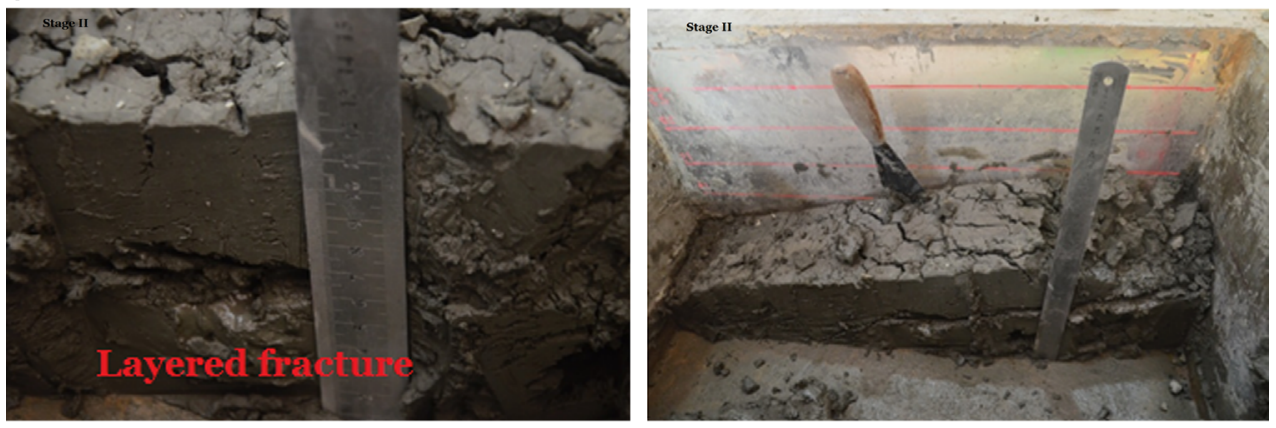

C

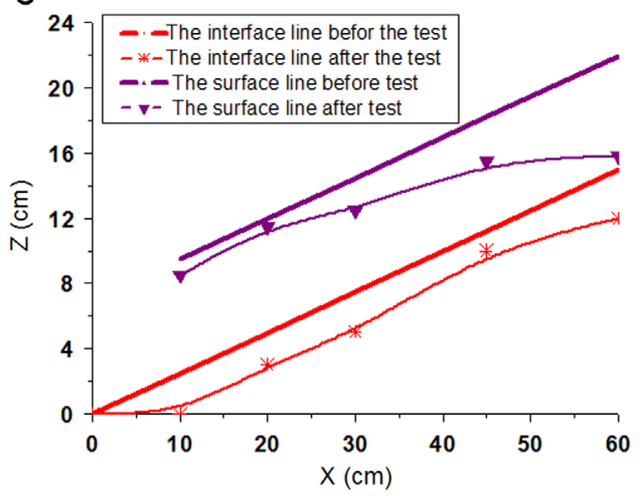

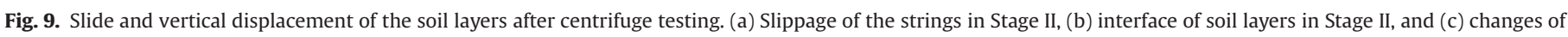
the thickness of hydrate layer and over layer.

there are layered fractures between the over layer and the hydrate layer where the shearing strength decreases greatly and gas and water are accumulated. From Fig. 9c, it can be seen that the whole hydrate layer was compressed, while the stratum at the toe uplifts due to the slide. The layered fracture and strings' inflection points and the changes of the two layers' thicknesses illustrates that the sliding surface is close to the interface between the over layer and the hydrate layer. It can be explained as follows: the hydrate dissociation and seepage causes the formation of layered fracture, the settlement and movement along the slope. The little changes of the over layer induces differences of the stiffness and strength between the over layer and the hydrate layer to be enlarged and so the displacement at the interface becomes discontinuous. Table 4 presents the density and water content of the over layer and the 
hydrate layer before and after the centrifuge test. The changes of densities and water contents could present a reference to the verification for the estimation of the seepage and consolidation.

\section{Discussions}

\subsection{Analysis of heat conduction}

When the heat source temperature is greater than the gasification temperature from liquid THF to gas THF and less than the boiling point of water, there will be two phase transformation fronts F1 and F2, three zones Z1, Z2, Z3 in THF hydrate-bearing silty soil as in Fig. 10a, where $\mathrm{Z1}$ represents the non-dissociated zone consisting of hydrate and skeleton; Z2 represents hydrate dissociation zone consisting of liquid, water and sediment skeleton; Z3 represents gasification zone consisting of gas, water and sediment skeleton; F1 represents the hydrate dissociation front; F2 represents the gasification front.

Solutions to this problem for each zone are as follows (Zhang et al., 2014):

In zone $\mathrm{Z} 1$ :

$\vartheta_{1}=\frac{\vartheta_{F 1}}{\operatorname{erf}\left(\frac{1}{2} \sqrt{\xi_{F 1}}\right)-1} \cdot \operatorname{erf}\left(\frac{1}{2} \sqrt{\xi}\right)-\frac{\vartheta_{F 1}}{\operatorname{erf}\left(\frac{1}{2} \sqrt{\xi_{F 1}}\right)-1}$

In zone $\mathrm{Z2}$ :

$\vartheta_{2}=\frac{\vartheta_{F 2}-\vartheta_{F 1}}{\operatorname{erf}\left(\frac{1}{2} \sqrt{\frac{\kappa_{1}}{\kappa_{2}} \xi_{F 2}}\right)-\operatorname{erf}\left(\frac{1}{2} \sqrt{\frac{\kappa_{1} \xi_{2}}{\kappa_{F 1}}}\right)} \cdot \operatorname{erf}\left(\frac{1}{2} \sqrt{\frac{\kappa_{1}}{\kappa_{2}} \xi}\right)$

Table 4

Density and water content of soil layers.

\begin{tabular}{lllllll}
\hline \multirow{2}{*}{$\begin{array}{l}\text { Basic } \\
\text { parameter }\end{array}$} & Over layer & & \multicolumn{2}{l}{ Hydrate layer } & & THF \\
\cline { 2 - 3 } & $\begin{array}{l}\text { Dry density } \\
\left(\mathrm{g} / \mathrm{cm}^{3}\right)\end{array}$ & $\begin{array}{l}\text { Water } \\
\text { content }(\%)\end{array}$ & $\begin{array}{l}\text { Dry density } \\
\left(\mathrm{g} / \mathrm{cm}^{3}\right)\end{array}$ & $\begin{array}{l}\text { Water } \\
\text { content }(\%)\end{array}$ & $\begin{array}{l}\text { Density } \\
\left(\mathrm{g} / \mathrm{cm}^{3}\right)\end{array}$ \\
\hline $\begin{array}{llllll}\text { Before testing } \\
\text { After testing }\end{array}$ & 1.3 & 34 & 1.1 & 56 & 0.8 \\
\hline
\end{tabular}

$$
+\left(\vartheta_{F 1}-\frac{\vartheta_{F 2}-\vartheta_{F 1}}{\operatorname{erf}\left(\frac{1}{2} \sqrt{\frac{\kappa_{1}}{\kappa_{2}} \xi_{F 2}}\right)-\operatorname{erf}\left(\frac{1}{2} \sqrt{\frac{\kappa_{1}}{\kappa_{2}} \xi_{F 1}}\right)} \cdot \operatorname{erf}\left(\frac{1}{2} \sqrt{\frac{\kappa_{1}}{\kappa_{2}} \xi_{F 1}}\right)\right)
$$

In zone Z3:

$\vartheta_{3}=\frac{\vartheta_{F 2}-1}{\operatorname{erf}\left(\frac{1}{2} \sqrt{\frac{\kappa_{1}}{\kappa_{3}} \xi_{F 2}}\right)} \cdot \operatorname{erf}\left(\frac{1}{2} \sqrt{\frac{\kappa_{1}}{\kappa_{3}}} \xi\right)+1$

where $\theta_{H}=T_{H}-T_{0}, \quad \theta_{e h}=T_{e h}-T_{0}, \quad \xi=\frac{x^{2}}{\kappa_{1} t}, \quad \vartheta=\frac{\theta}{\theta_{H}}, \quad \xi_{F 1}$ and $\xi_{F 2}$ represent the self-similar parameters for fronts F1 and F2, $\vartheta_{1}, \vartheta_{2}, \vartheta_{3}$ and $\kappa_{1}, \kappa_{2}, \kappa_{3}$ represent dimensionless temperature and thermal diffusion coefficient in $\mathrm{Z} 1, \mathrm{Z2}, \mathrm{Z3}$, respectively. $\xi_{F 1}$ and $\xi_{F 2}$ can be obtained by a Newtonian iterative method; the expansions of transformation fronts $\mathrm{F} 1$ and $\mathrm{F} 2$ can be expressed as $X_{F 1}=\left(\xi_{F 1} \kappa_{1} t\right)^{1 / 2}$

and $X_{F 2}=\left(\xi_{F 2} \kappa_{1} t\right)^{1 / 2}$, and the temperature fields can be obtained at the same time.

The hydrate dissociation zone and the temperature evolution were simulated through above equations. The measured and simulated results at the place of $\mathrm{T} 2$ were in agreement with each other (Fig. 10b and c).

\subsection{Analysis of the seepage and consolidation}

Theoretically, the soil stress (self-weight of the soil) in prototype can be calculated by $\sigma_{m}=\rho \cdot N \cdot g \cdot h$. So the soil stress was

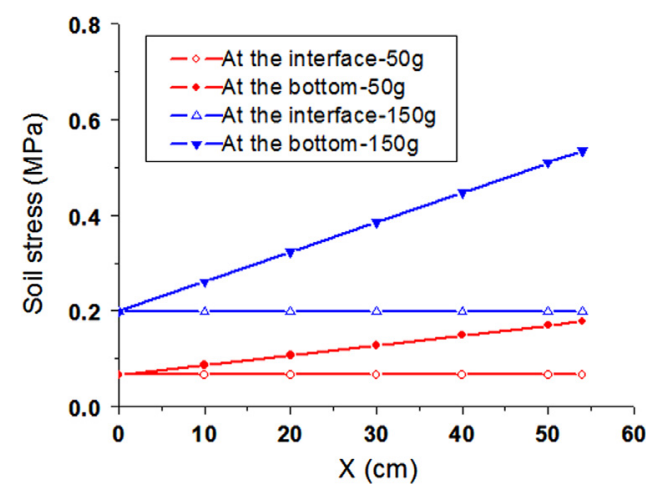

Fig. 11. Stress distribution at the interface and bottom of soil layers.
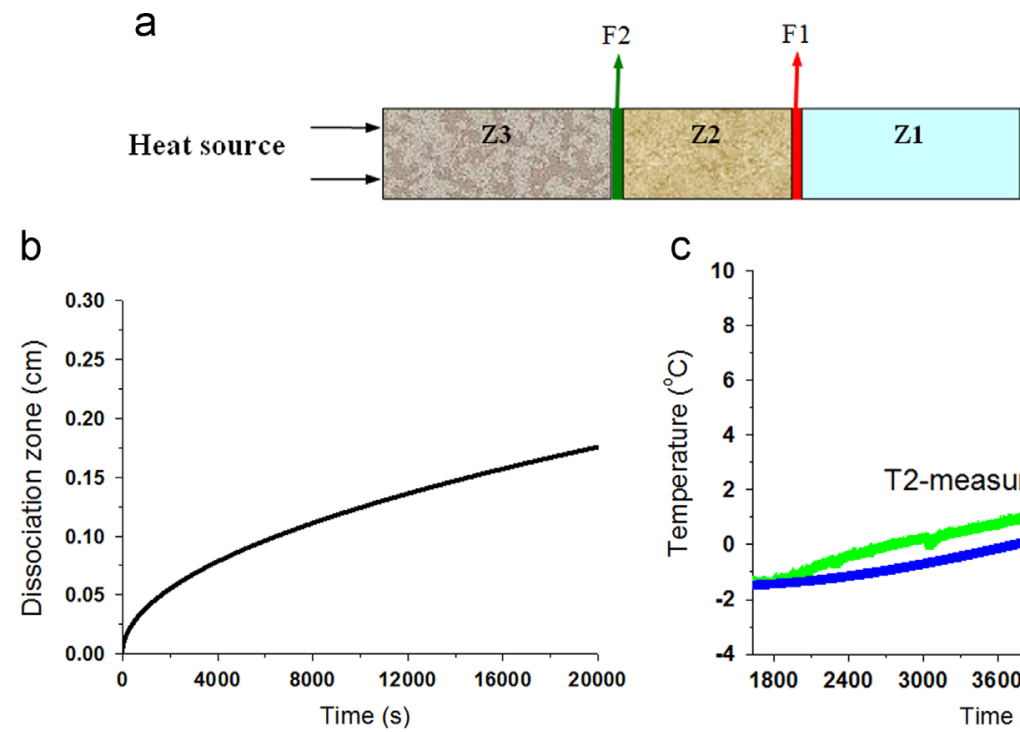

\footnotetext{
C
}

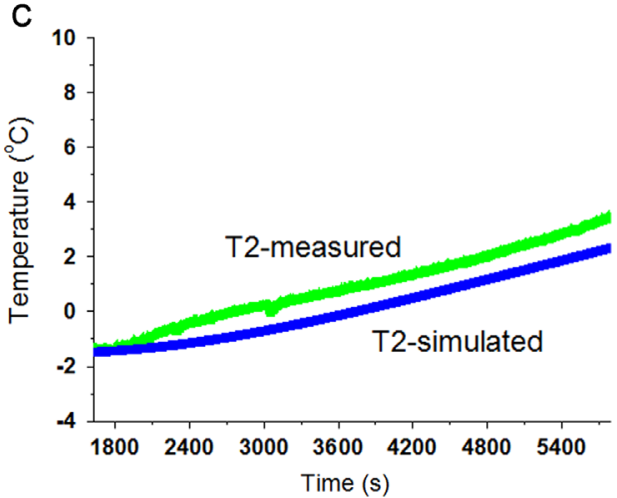

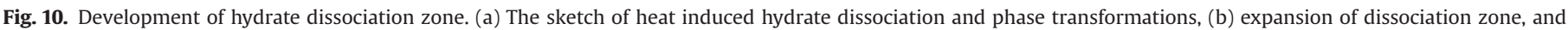
(c) temperature evolution. 

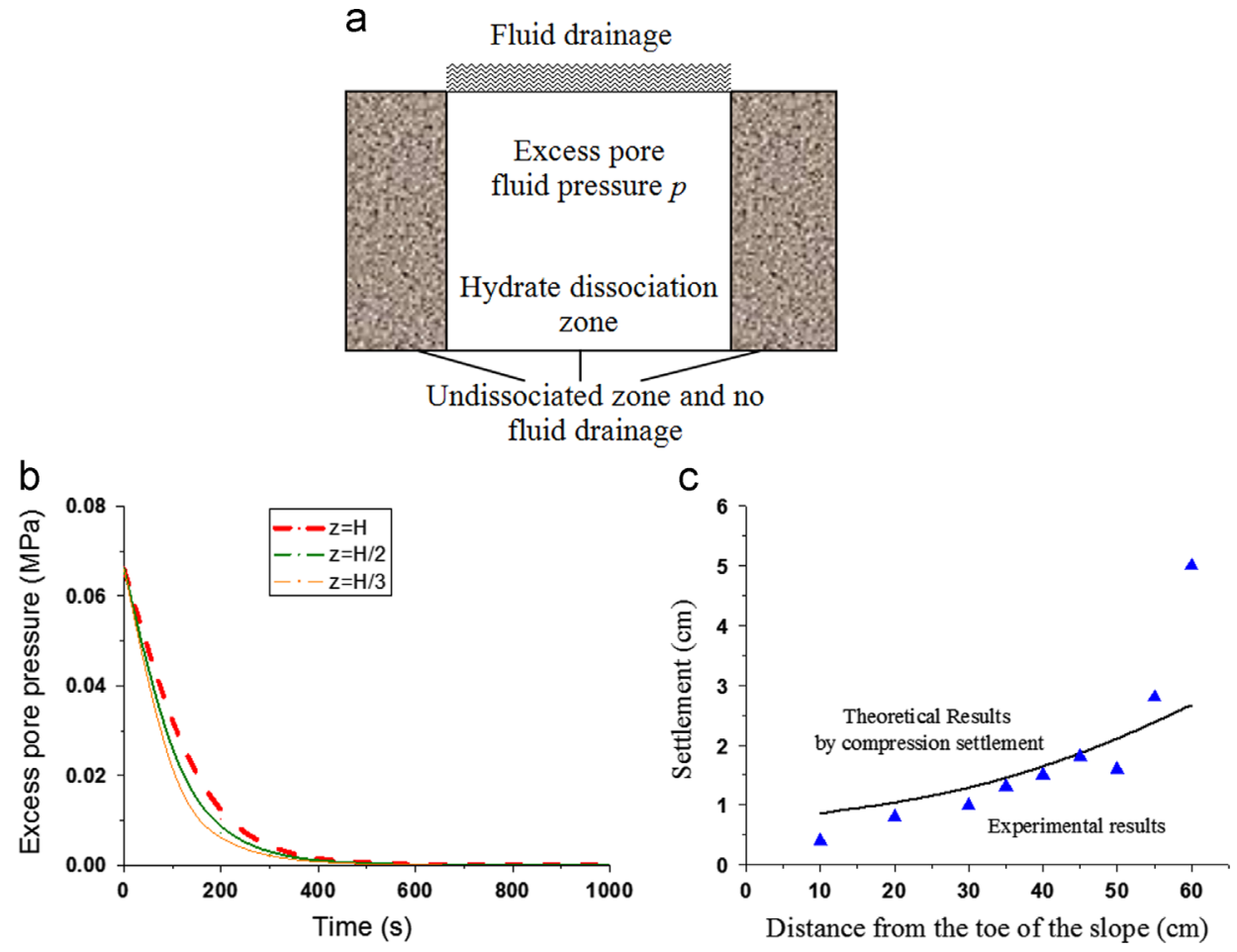

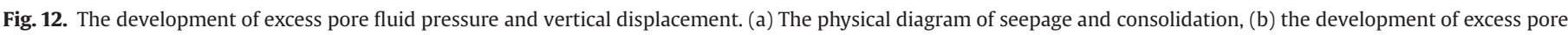
fluid pressure, and (c) vertical displacement after hydrate dissociation.

$0.06 \mathrm{MPa}$ at the interface of hydrate layer and over layer and 0.06-0.19 MPa at the bottom of the hydrate layer in Stage I. And the soil stress was $0.18 \mathrm{MPa}$ at the interface and $0.18-0.57 \mathrm{MPa}$ at the bottom of the hydrate layer in Stage II (Fig. 11). In the two stages, the pore fluid pressure even exceeded the over the soil stress.

Zhang et al. (2011) measured the pore pressure development during THF hydrate dissociation in sediments. When the hydrate dissociates at $70{ }^{\circ} \mathrm{C}$, the pore pressure is $0.125 \mathrm{MPa}$, which is $0.065 \mathrm{MPa}$ larger than the pressure of the over layer. Assume the pore fluid in the hydrate dissociation zone could be drained through the over layer (Fractures and side seepage path between model box and over layer). The physical process of seepage and consolidation is illustrated in Fig. 12a, according to the consolidation theory, the development of excess pore fluid pressure can be shown as follows (Craig, 1983):

$p_{z, t}=\frac{4 p}{\pi} \sum_{m=1}^{\infty} \frac{1}{m} \sin \frac{m \pi z}{2 H} \exp \left(-\frac{\pi^{2} m^{2}}{4} T_{v}\right), m=1,3,5, \cdots$

where $T_{v}=\frac{C_{v}}{H^{2}} t, C_{v}=\frac{k\left(1+e_{0}\right)}{a \gamma_{w}}, k$ is the permeability coefficient, $e_{0}$ is the initial void ratio, $a$ is the compression coefficient, and $\gamma_{w}$ is the specific gravity.

The final vertical displacement by compression can be obtained:

$S=\frac{p H}{E_{S}}$

From Fig. 12b, the excess pore pressure dissipates much faster relative to heat conduction in a same geometric size, and the pressure measured in the tests could keep stable. The experimental results are close with the theoretical results at the middle of the slope, smaller at the bottom and larger at the top (Fig. 12c). The main reason is that the instability of the slope is determined by two factors: one was vertical displacement by seepage and consolidation, the other one was the sliding between hydrate layer and over layer.

\section{Conclusions}

Through centrifuge test, the evolution and physical mechanism of stratum instability were investigated during/after hydrate dissociation. The expansion of cracks, settling zone and the slippage between over layer and hydrate layer was observed.

The mechanism of the stratum instability can be described as: heat transfer leads to the phase transformation of hydrate and expansion of hydrate dissociation zone and accordingly the softening of the soil layer accompanying the seepage and consolidation in hydrate dissociation zone. The layered fracture filled by water or gas between the hydrate dissociation zone and the over layer decreases the shearing strength, and slippage occurs when the dissociation zone expands to some certain extent.

The sliding occurred gradually accompanying the formation of tensile failures in the over layer and the layered fracture between the hydrate layer and the over layer in the centrifuge test.

The characteristic time of heat transfer was 100 times that of seepage and consolidation, and so the decoupling method was used in analysis. By comparison of temperature evolution and excess pore fluid pressure dissipation with test results, the method was shown to be valid. This research is expected to provide verification to further numerical simulations and evaluation of strata instability due to hydrate dissociation.

\section{Acknowledgments}

This study is part of Project no. GZH201100311 funded by China Geological Survey and Projects nos. 51239010, 11102209, and 41376078 funded by National Natural Science Foundation of China.

\section{References}

Craig, R. F., 1983. Soil mechanics. Third ed., Van Nostrand Reinhold Co. Ltd., Wokingham, Berkshire, England. 
Jones, K.W., Kerkar, P.B., Mahajan, D., Lindquist, W.B., Feng, H., 2007. Microstructure of natural hydrate host sediments. Nucl. Instrum. Methods Phys. Res. B 261 (1-2), 504-507.

Hong, H., Pooladi-darvish, M., Bishnoi, P.R., 2003. Analytical modeling of gas production from hydrates in porous media. J. Can. Pet. Technol. 42 (11), 45-56.

Kvenvolden, K.A., Lorenson, T.D., 2001. The global occurrence of natural gas hydrate. Geophys. Monogr. 124, 3-18.

Kwon, T.H., Cho, G.C., Santamarina, J.C., 2008. Gas hydrate dissociation in sediments: pressure-temperature evolution. Geochem. Geophys. Geosyst. 9, Q03019. http://dx.doi.org/10.1029/2007GC001920.

Kwon, T.H., Oh, T.M., Choo, Y.W., et al., 2013. Geomechanical and thermal responses of hydrate bearing sediments subjected to thermal simulation: physical modeling using a geotechnical centrifuge. Energy Fuels 27, 4507-4522.

Kimoto, S., Oka, F., Fushita, T., 2010. A chemo-thermo-mechanically coupled analysis of ground deformation induced by gas hydrate dissociation. Int. J. Mech. Sci. 52, 365-376.

Klar, A., Soga, K., Ng, M.Y.A., 2010. Coupled deformation-flow analysis for methane hydrate extraction. Geotechnique 60 (10), 765-776.

Lee, J.Y., Yun, T.S., Santamarina, J.C., Ruppel, C., 2007. Observations related to tetrahydrofuran and methane hydrates for laboratory studies of hydratebearing sediments. Geochem. Geophy. Geosyst. 8 (6), Q06003.

Li, G., Moridis, G.J., Zhang, K.N., Li, X.S., 2010. Evaluation of gas production potential from marine gas hydrate deposits in Shenhu area of South China sea. Energy Fuels 24 (11), 6018-6033.

Lu, X.B., Wang, L., Wang, S.Y., et al., 2008. Study on the mechanical properties of Tetrahydrofuran hydrate deposit. In: Proceedings of the 18th InternationalOffshore and Polar Engineering Conference. pp. 57-60.

Lu, X.B., Zhang, X.H., Hu, K.H., 2013. Centrifugal experiment on stratum instability and failure process due to gas hydrate dissociation, Global View of Engineering Geology and the Environment-Wu \& Qi (eds.). Taylor \& Francis Group, London, pp. 197-201.

Sloan, E.D., 1998. Clathrate Hydrates of Natural Gases. Marcel Dekker Inc., New York.
Sultan, N., et al., 2004a. Dynamics of gas hydrate: case of Congo continental slope. Mar. Geol. 206, 1-18.

Sultan, N., et al., 2004b. Effect of gas hydrates melting on seafloor slope instability. Mar. Geol. 213, 379-401.

Taylor, 1995. Geotechnical Centrifuge Technology. Blackie Academic, London.

Tan, Q.M., 2011. Dimensional Analysis: With Case Studies In Mechanics, Berlin, Heidelberg. Springer-Verlag, Berlin Heidelberg.

Waite, W.F., et al., 2009. Physical properties of hydrate-bearing sediments. Rev. Geophy. 47, 1-38, RG4003/2009.

Winters, W.J., Pecher, I.A., Waite, W.F., Mason, D.H., 2004. Physical properties and rock physics models of sediment containing natural and laboratory-formed methane gas hydrate. Am. Mineral. 89, 1221-1227.

Winters, W.J., Waite, W.F., Mason, D.H., et al., 2007. Methane gas hydrate effect on sediment acoustic and strength properties. J. Pet. Sci. Eng. 56, 127-135.

Wang S.Y., Zheng W., Lu X.B., 2009. The effects of gas hydrate dissociation on the stability of pipeline in seabed. In: Proceedings of the 19th InternationalOffshore and Polar Engineering Conference, Japan. pp. 49-53.

$\mathrm{Xu}, \mathrm{W}$., Germanovich, L.N., 2006. Excess pore pressure resulting from methane hydrate dissociation in marine sediments: a theoretical approach. J. Geophys. Res. 111, B011104. http://dx.doi.org/10.1029/2004JB003600.

Xu, W., Germanovich, L.N., 2007. Reply to comment by Nabil Sultan on "Excess pore pressure resulting from methane hydrate dissociation in marine sediments: a theoretical approach". J. Geophys. Res. 112, B02104. http://dx.doi.org/10.1029/ 2006JB004722.

Yun, T.S., Santamarina, J.C., Ruppel, C., 2007. Mechanical properties of sand, silt, and clay containing tetrahydrofuran hydrate. J. Geophy. Res. 112, B04106.

Zhang, X.H., Lu, X.B., Li, Q.P., 2011. Formation of layered fracture and outburst during gas hydrate dissociation. J. Pet. Sci. Eng. 76 (3-4), 212-216.

Zhang, X.H., Lu, X.B., Zheng, Z.M., 2014. Heat-induced evolution of phase transformations in tetrahydrofuran hydrate-bearing sediment. J. Heat Transf. 136 (5), 052002.

Zhu, Y.H., Zhang, Y.Q., Wen, H.J., et al., 2010. Gas hydrates in the qilian mountain permafrost, Qinghai, Northwest China. Acta Geol. Sin. 84 (1), 1-10. 